\section{Retrospective study of occurrence and types of complications associated with different procedures to correct traumatic hip luxation in 44 dogs}

\section{Aaron Lutchman, Pilar Lafuente}

Department of Clinical Science and Services, The Royal Veterinary College, Hawkshead Lane, North Mymms, Hatfield, Hertfordshire, AL9 7TA, Hatfield, United Kingdom

\section{OBJECTIVES}

Retrospective, observational and analytical study assessing the demography of traumatic hip luxation in dogs and the complications associated with different correction techniques. This study aims to assess whether certain techniques are superior with regard to outcomes, associated complications and the respective complication rate.

\section{METHODS}

The cases of animals with unilateral or bilateral luxation of the coxo-femoral joint as a result of trauma between 2009 and 2018 were reviewed. Signalment, clinical signs, treatment type (closed reduction, closed reduction followed by surgical reduction and stabilization, or immediate surgical reduction and stabilization), outcome and complications were recorded for each patient. Statistical evaluation was performed to identify relationships between treatment type and outcome or rate of complications.

\section{RESULTS}

Forty-four dogs were included in the study. Mean age was 4 years (range 3 months -12 years) with a mean weight of $19 \mathrm{~kg}$ (range $4-48 \mathrm{~kg}$ ). Road traffic accidents ( $n=17)$ and animals exercising ( $n=8)$ were the most common causes of traumatic luxation. Seven surgical procedures were represented in this data set, the most common being ilio-femoral suture. Closed reduction was attempted primarily in 33/44 cases however a significant proportion of these cases re-luxated ( $n=24)$, requiring further surgical correction. Complication of re-luxation post-surgical reduction was notably less $(n=4)$. Post surgical infection was uncommon, recorded in $(n=2)$ cases

\section{STATEMENT (CONCLUSIONS)}

Given the increased rate of re-luxation in animals managed with closed reduction it could be suggested that surgical intervention is warranted as the primary course of treatment.
The effectiveness of marine based fatty acid compound (pcso-524) alone and combined with previcox in the treatment of canine osteoarthritis

\section{Brian Beale}

Gulf Coast Veterinary Specialists, Houston, USA

\section{OBJECTIVES}

The purpose of this study is to assess the effectiveness of a marine-based fatty acid compound alone and in combination with firocoxib for treatment of osteoarthritisassociated pain in dogs using objective measures of limb use and validated subjective assessments.

\section{METHODS}

A double-blinded randomized prospective clinical trial was performed with 31 dogs. Dogs were randomly allocated to a PCSO-524 group (PCSO) or a Firocoxib + PCSO-524 (FCX-PCSO) group. Owners were masked to use of firocoxib by using identical placebo tablets in the PCSO-524 group. Force plate gait analysis and the owner-completed Canine Brief Pain Inventory toolwere used to evaluate patients at 0,2 and 4 weeks. Data were analyzed using repeated measurement analysis with significant level set a $5 \%(\alpha=0.05)$.

\section{RESULTS}

Peak Vertical Force (PVF) values were significantly increased over baseline at week 2 and week 4 in both groups $(p<0.05)$. A significant decrease in the CBPI scores (improvement) was seen at week 2 and 4 in the PCSO 